\title{
The prognostic value of the systemic inflammatory score in patients with unresectable metastatic colorectal cancer
}

\author{
MASATSUNE SHIBUTANI, KIYOSHI MAEDA, HISASHI NAGAHARA, TATSUNARI FUKUOKA, \\ SHINJI MATSUTANI, KENJIRO KIMURA, RYOSUKE AMANO, KOSEI HIRAKAWA and MASAICHI OHIRA
}

Department of Surgical Oncology, Osaka City University Graduate School of Medicine, Osaka 545-8585, Japan

Received October 19, 2017; Accepted April 26, 2018

DOI: $10.3892 / \mathrm{ol} .2018 .8628$

\begin{abstract}
Inflammation has been widely recognized as a contributor to cancer progression and several inflammatory markers have been reported as associated with the clinical outcomes in patients with various types of cancer. Recently, a novel inflammatory marker, the systemic inflammatory score (SIS), which is based on a combination of the lymphocyte-to-monocyte ratio (LMR) and the serum albumin concentration has been reported as a useful prognostic marker. The aim of the present study was to assess the prognostic value of the SIS in patients with unresectable metastatic colorectal cancer (mCRC). The retrospective cohort study included 160 patients who underwent combination chemotherapy for unresectable mCRC between January 2008 and December 2016. The SIS was used to classify the patients into three groups based on their LMR and the serum albumin concentration. Patients with high-LMR and high serum albumin level were given a score of 0 ; patients with low-LMR or low serum albumin level were given a score of 1 ; patients with low-LMR and low serum albumin level were given a score of 2 . There were significant differences in the overall survival among the three SIS groups and the SIS was an independent prognostic factor for the overall survival. Although the SIS was significantly associated with the overall survival rate even when using the original cut-off values, the SIS according to the new cut-off values had a more accurate prognostic value. The present study determined that the SIS was a useful biomarker for predicting the survival outcomes in patients with unresectable $\mathrm{mCRC}$, although the optimum cut-off
\end{abstract}

Correspondence to: Dr Masatsune Shibutani, Department of Surgical Oncology, Osaka City University Graduate School of Medicine, 1-4-3 Asahi-machi Abeno-ku, Osaka 545-8585, Japan E-mail: fbxbj429@ybb.ne.jp

Abbreviations: SIS, systemic inflammatory score; LMR, lymphocyte-to-monocyte ratio; mCRC, metastatic colorectal cancer; NLR, neutrophil-to-lymphocyte ratio; GPS, Glasgow prognostic score; PS, performance status; ROC curve, receiver operating characteristic curve; CI, confidence interval

Key words: metastatic colorectal cancer, prognostic marker, systemic inflammatory score, chemotherapy, survival value of the SIS according to the patients' background needs to be examined in further studies.

\section{Introduction}

Inflammation is known to contribute to cancer progression $(1,2)$, and several inflammatory markers, such as the neutrophil-to-lymphocyte ratio (NLR), the lymphocyte-to-monocyte ratio (LMR) and the Glasgow prognostic score (GPS) have been reported to be associated with clinical outcomes in patients with various types of cancer, including colorectal cancer (3-8). Recently, a new inflammatory marker, the systemic inflammatory score (SIS), based on the combination of the LMR and the serum albumin concentration has been reported to be a useful prognostic marker in patients with clear-cell renal cell carcinoma, colorectal cancer and oral cavity squamous cell carcinoma (9-11).

However, there are only a few reports on the SIS, and the prognostic value of the SIS in patients with unresectable metastatic colorectal cancer (mCRC) remains unclear. In addition, the optimum cut-off value may change depending on the type of cancer and stage and merits further study.

This study aimed to evaluate the prognostic value of the SIS and to determine its optimum cut-off value in patients with unresectable $\mathrm{mCRC}$ who underwent chemotherapy.

\section{Materials and methods}

Patients. This retrospective cohort study included 160 patients who underwent combination chemotherapy for unresectable mCRC at the Department of Surgical Oncology of Osaka City University (Osaka, Japan) between January 2008 and December 2016.

Methods. Blood samples were collected within one week prior to the initiation of chemotherapy. We analyzed the differential white blood cell count using an XE-5000 hematology analyzer (Sysmex, Kobe, Japan) based on the manufacturer's protocol. The LMR was calculated by dividing the absolute number of circulating lymphocytes by the absolute number of circulating monocytes. We assessed the serum albumin concentrations by a chemiluminescent immunoassay (Wako, Osaka, Japan) according to the manufacturer's protocol. The SIS was defined according to the methods of a previous report (9), using the 
combination of the LMR and the serum albumin concentration: patients with LMR $>4.44$ and serum albumin level $>4.0 \mathrm{~g} / \mathrm{dl}$ were given a score of 0 ; patients with $\mathrm{LMR} \leq 4.44$ or serum albumin level $\leq 4.0 \mathrm{~g} / \mathrm{dl}$ were given a score of 1 ; patients with $\mathrm{LMR} \leq 4.4$ and 4 serum albumin level $\leq 4.0 \mathrm{~g} / \mathrm{dl}$ were given a score of 2 . The location of the primary tumor was defined as follows. The oral side of the splenic flexure was termed 'the right side' and the anal side of splenic flexure was termed 'the left side'. Furthermore, we defined synchronous and metachronous metastases as follows. Synchronous metastases were defined as metastatic lesions that were already confirmed at the time of the diagnosis of the primary lesion; metachronous metastases were defined as metastatic lesions that developed after the excision of the primary tumor, regardless of the period.

Ethical considerations. All patients were informed of the investigational nature of this study and provided their written informed consent for the retrospective analysis of their data. Full ethical approval was granted by the Ethics Committee of Osaka City University (approval no. 926).

Statistical analysis. The significance of the correlations between the SIS and the clinicopathological characteristics were analyzed using the Chi-squared test. Survival curves were constructed using the Kaplan-Meier method and were compared using the log-rank test. A multivariate analysis was performed using a Cox proportional hazards model. All of the statistical analyses were performed using the SPSS software program (version 19.0; IBM, Armonk, NY, USA). P<0.05 was considered to indicate a statistically significant difference.

\section{Results}

Patients' baseline characteristics. The characteristics of the 160 patients in the present study are summarized in Table I. The study population included 86 male patients and 74 female patients. The median age of the patients was 65 years (range: 18 to 89). According to the definition of the Eastern Cooperative Oncology Group performance status (PS), 140 patients were classified as having a PS of 0,17 were classified as having a PS of 1, and 3 were classified as having a PS of 2. A total of 39 patients had primary tumors located on the right side, and 121 had primary tumors located on the left side. One hundred and seven patients had single-organ metastasis, and 53 multiple organs affected by metastases. All of the patients underwent combination chemotherapy with oxaliplatin, irinotecan plus 5-fluorouracil/leucovorin, or a prodrug of 5-fluorouracil as first-line chemotherapy. The regimens used for all of the patients in this study were considered to have the same efficacy (12-14). Seventy-five patients received FOLFOX, 53 received CapeOX, 25 received FOLFIRI, and 7 SOX. A total of 103 patients underwent chemotherapy combined with molecular-targeted therapy. The median follow-up period for the surviving patients was 21.8 months (range: 1.2 to 94.0 months). A total of 113 patients died during the follow-up period.

Correlations between the SIS and clinicopathological factors. The correlations between the SIS and clinicopathological factors are shown in Table II. The SIS and clinicopathological factors did not differ to a statistically significant extent.
Table I. The patients' baseline characteristics.

\begin{tabular}{|c|c|}
\hline Characteristics & No. of patients \\
\hline Median age, years (range) & $65(18-89)$ \\
\hline \multicolumn{2}{|l|}{ Sex } \\
\hline Male & 86 \\
\hline Female & 74 \\
\hline \multicolumn{2}{|l|}{ Performance status } \\
\hline 0 & 140 \\
\hline 1 & 17 \\
\hline 2 & 3 \\
\hline \multicolumn{2}{|l|}{ Location of primary tumor } \\
\hline Right side & 39 \\
\hline Left side & 121 \\
\hline \multicolumn{2}{|l|}{ Histological type } \\
\hline Well, moderately & 143 \\
\hline Poorly, mucinous & 17 \\
\hline \multicolumn{2}{|l|}{ RAS status } \\
\hline Wild type & 64 \\
\hline Mutant type & 54 \\
\hline Unknown & 42 \\
\hline \multicolumn{2}{|c|}{ Detection of unresectable tumor } \\
\hline Synchronous & 106 \\
\hline Metachronous & 54 \\
\hline \multicolumn{2}{|c|}{ Number of organs affected by metastasis } \\
\hline One organ & 107 \\
\hline Multiple organs & 53 \\
\hline \multicolumn{2}{|l|}{ Peritoneal dissemination } \\
\hline Negative & 125 \\
\hline Positive & 35 \\
\hline \multicolumn{2}{|c|}{ First-line chemotherapy regimen } \\
\hline FOLFOX & 75 \\
\hline CapeOX & 53 \\
\hline FOLFIRI & 25 \\
\hline SOX & 7 \\
\hline \multicolumn{2}{|l|}{ Molecular-targeted therapy } \\
\hline Bevacizumab & 85 \\
\hline Cetuximab & 11 \\
\hline Panitumumab & 7 \\
\hline None & 57 \\
\hline
\end{tabular}

Lymphocyte-to-monocyte ratio

Median (range)

$4.53(1.25-14.06)$

Serum albumin concentration

Median (range)

$3.9(2.5-4.9)$

Systemic inflammatory score

0

46

$1 \quad 68$

2

46

Prognostic significance of the SIS according to the original cut-off values defined in the previous report. The median overall survival time was 31.6 months in those with a SIS of 
Table II. The correlations between the SIS and the clinicopathological factors.

\begin{tabular}{|c|c|c|c|c|}
\hline \multirow[b]{2}{*}{ Clinicopathological factor } & \multicolumn{3}{|c|}{ SIS } & \multirow[b]{2}{*}{ P-value } \\
\hline & $0(n=46)$ & $1(n=68)$ & $2(n=46)$ & \\
\hline Age & & & & 0.385 \\
\hline$<65$ years & 27 & 31 & 24 & \\
\hline$\geq 65$ years & 19 & 37 & 22 & \\
\hline Sex & & & & 0.718 \\
\hline Male & 23 & 39 & 24 & \\
\hline Female & 23 & 29 & 22 & \\
\hline Location of primary tumor & & & & 0.416 \\
\hline Right side & 13 & 18 & 8 & \\
\hline Left side & 33 & 50 & 38 & \\
\hline Performance status & & & & 0.630 \\
\hline 0 & 42 & 58 & 40 & \\
\hline$\geq 1$ & 4 & 10 & 6 & \\
\hline Histological type & & & & 0.335 \\
\hline Well, moderately & 43 & 58 & 42 & \\
\hline Poorly, mucinous & 3 & 10 & 6 & \\
\hline RAS status & & & & 0.310 \\
\hline Wild type & 25 & 22 & 17 & \\
\hline Mutant type & 16 & 26 & 12 & \\
\hline Unknown & 5 & 20 & 17 & \\
\hline Detection of unresectable tumor & & & & 0.119 \\
\hline Synchronous & 29 & 41 & 36 & \\
\hline Metachronous & 17 & 27 & 10 & \\
\hline Number of organs affected by metastasis & & & & 0.960 \\
\hline One organ & 30 & 46 & 31 & \\
\hline Multiple organs & 16 & 22 & 15 & \\
\hline Peritoneal dissemination & & & & 0.101 \\
\hline Negative & 34 & 50 & 41 & \\
\hline Positive & 12 & 18 & 5 & \\
\hline Molecular-targeted therapy & & & & 0.312 \\
\hline Absent & 13 & 24 & 20 & \\
\hline Present & 33 & 44 & 26 & \\
\hline
\end{tabular}

SIS, systemic inflammatory score.

0, 22.9 months in those with a SIS of 1 , and 20.6 months in those with a SIS of 2. A log-rank test demonstrated significant differences in the overall survival among the three groups $(\mathrm{P}=0.0021)$. However, there were no significant differences in the overall survival between the patients with a SIS of 1 and those with a SIS of 2, although the overall survival rate tended to be worse in patients with a SIS of 2 than in those with a SIS of 1 ( $\mathrm{P}=0.0810$; Fig. 1$)$.

Univariate and multivariate analyses of the risk factors for overall survival. In the univariate analysis, the PS, the location of the primary tumor, the RAS status and the SIS were associated with overall survival. Furthermore, a multivariate analysis demonstrated that gender, the location of the primary tumor and the SIS were independent prognostic factors for survival (Table III).

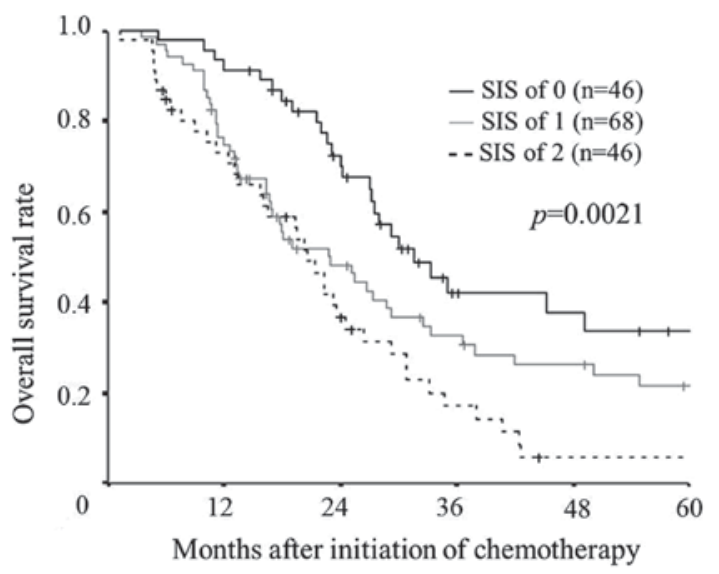

Figure 1. Kaplan-Meier survival curves for the overall survival according to the SIS based on the original cut-off values. SIS, systemic inflammatory score. 
Table III. Univariate and multivariate analyses of risk factors for overall survival.

\begin{tabular}{|c|c|c|c|c|c|c|}
\hline \multirow[b]{2}{*}{ Characteristic } & \multicolumn{3}{|c|}{ Univariate analysis } & \multicolumn{3}{|c|}{ Multivariate analysis } \\
\hline & Hazard ratio & $95 \% \mathrm{CI}$ & P-value & Hazard ratio & $95 \% \mathrm{CI}$ & P-value \\
\hline \multicolumn{7}{|l|}{ Age (years) } \\
\hline$<65$ & Reference & & & Reference & & \\
\hline$\geq 65$ & 1.318 & $0.908-1.912$ & 0.146 & 1.309 & $0.825-2.077$ & 0.254 \\
\hline \multicolumn{7}{|l|}{ Sex } \\
\hline Male & Reference & & & Reference & & \\
\hline Female & 1.256 & $0.868-1.817$ & 0.227 & 1.771 & $1.097-2.859$ & 0.019 \\
\hline \multicolumn{7}{|l|}{ Performance status } \\
\hline 0 & Reference & & & Reference & & \\
\hline$\geq 1$ & 1.824 & $1.085-3.065$ & 0.023 & 1.448 & $0.664-3.154$ & 0.352 \\
\hline \multicolumn{7}{|c|}{ Location of primary tumor } \\
\hline Left side & Reference & & & Reference & & \\
\hline Right side & 1.982 & $1.273-3.086$ & 0.002 & 2.179 & $1.192-3.984$ & 0.011 \\
\hline \multicolumn{7}{|l|}{ Histological type } \\
\hline Well, moderately & Reference & & & Reference & & \\
\hline Poorly, mucinous & 0.685 & $0.355-1.321$ & 0.259 & 0.552 & $0.257-1.185$ & 0.127 \\
\hline \multicolumn{7}{|l|}{ RAS status } \\
\hline Wild type & Reference & & & Reference & & \\
\hline Mutant type & 1.699 & $1.100-2.625$ & 0.017 & 1.533 & $0.957-2.456$ & 0.075 \\
\hline \multicolumn{7}{|c|}{ Detection of unresectable tumor } \\
\hline Synchronous & Reference & & & Reference & & \\
\hline Metachronous & 1.107 & $0.740-1.656$ & 0.621 & 1.171 & $0.642-2.133$ & 0.607 \\
\hline \multicolumn{7}{|c|}{ The number of organs affected by metastasis } \\
\hline 1 & Reference & & & Reference & & \\
\hline$\geq 2$ & 1.119 & $0.756-1.656$ & 0.573 & 0.650 & $0.360-1.175$ & 0.154 \\
\hline \multicolumn{7}{|c|}{ Peritoneal dissemination } \\
\hline Negative & Reference & & & Reference & & \\
\hline Positive & 1.145 & $0.726-1.805$ & 0.560 & 1.739 & $0.850-3.559$ & 0.130 \\
\hline \multicolumn{7}{|c|}{ Molecular targeted therapy } \\
\hline Present & Reference & & & Reference & & \\
\hline Absent & 1.116 & $0.764-1.630$ & 0.571 & 0.960 & $0.601-1.534$ & 0.866 \\
\hline \multicolumn{7}{|l|}{ SIS } \\
\hline 0 & Reference & & & Reference & & \\
\hline 1 & 1.567 & $0.975-2.516$ & 0.063 & 1.873 & $1.075-3.263$ & 0.027 \\
\hline 2 & 2.386 & $1.451-3.921$ & 0.001 & 4.138 & $2.163-7.916$ & $<0.001$ \\
\hline
\end{tabular}

CI, confidence interval; SIS, systemic inflammatory score.

Setting new cut-off values for the LMR and the serum albumin concentration. A receiver operating characteristic (ROC) curve analysis was used to determine the optimal cut-off LMR and serum albumin level. We used the LMR and serum albumin level, continuous variables, as the test variable and the 24.4-month survival (median survival time: 24.4 months) as the state variable. The optimum cut-off values were selected based on the highest Youden index; the optimum cut-off LMR was 2.96 (sensitivity: $89.4 \%$; specificity: $35.1 \%$ ), while the optimum cut-off serum albumin level was 4.0 (sensitivity: 53.0\%; specificity: $73.4 \%$ ) (Fig. 2). The patients were classified into the high-LMR $(\mathrm{n}=120)$ and low-LMR $(\mathrm{n}=40)$ groups based on the new cut-off LMR. In the same way, the patients were classified into the high-ALB $(n=60)$ and low-ALB $(n=100)$ groups.

Prognostic value of the LMR and the serum albumin concentration. The patients in the low-LMR group had a significantly worse overall survival rate in comparison to the patients in the high-LMR group $(\mathrm{P}<0.0001$; Fig. 3A). Similarly, the patients in the low-ALB group had significantly worse overall survival in comparison to the high-ALB group ( $\mathrm{P}=0.0004$; Fig. 3B). 

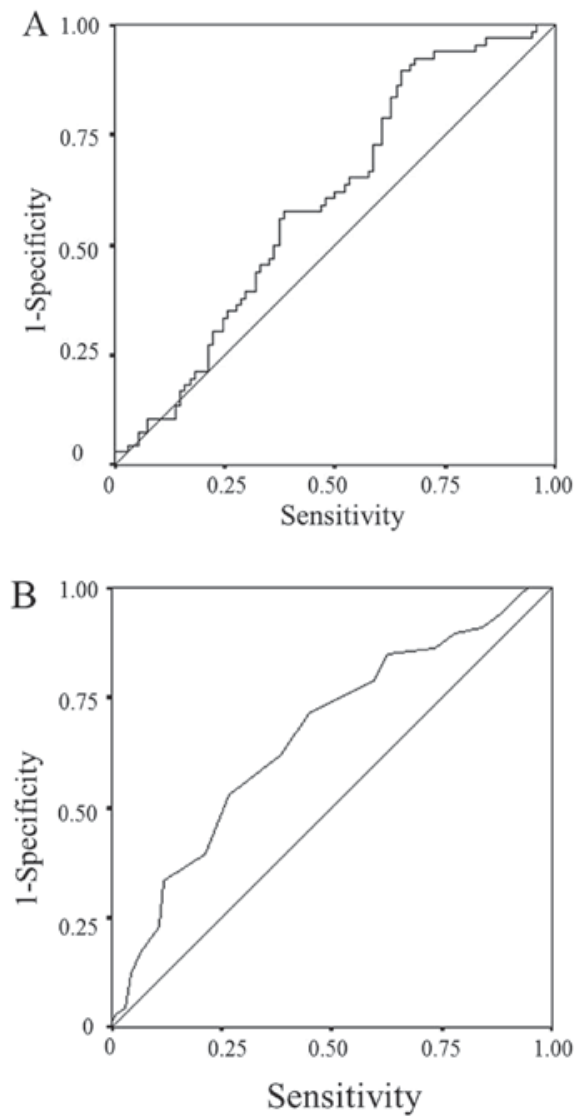

Figure 2. (A) The ROC curve for the lymphocyte-to-monocyte ratio. Area under the curve, $0.599 ; 95 \%$ confidence interval, $0.512-0.750 ; \mathrm{P}=0.033$. (B) The ROC curve for the serum albumin concentration. Area under the curve, 0.664; 95\% confidence interval, 0.579-0.750; $\mathrm{P}<0.001$. ROC, receiver operating characteristic.

Prognostic value of the SIS according to the new cut-off value derived in our data set. According to the new cut-off value (LMR: 2.96, serum albumin level: 4.0) as well as the original cut-off value, the SIS was significantly associated with the overall survival rates $(\mathrm{P}<0.0001$; Fig. 4). Furthermore, there were significant differences between the each subgroup.

\section{Discussion}

The results obtained in this study suggested that the SIS was significantly associated with the survival outcomes and may be useful as a prognostic biomarker in patients with unresectable mCRC. To our knowledge, this is the first study to assess the prognostic value of the SIS in patients with unresectable mCRC.

Albumin is a protein synthesized in the liver. Under conditions of systemic inflammation, the ability to synthesize albumin decreases, resulting in hypoalbuminemia (15). Therefore, a low serum albumin concentration is associated with ongoing systemic inflammation. Due to the fact that continuous systemic inflammation promotes cancer progression $(1,2)$, hypoalbuminemia is associated with a poor survival (16).

The LMR reflects the balance between the immune status of the host and the degree of tumor burden. Lymphocytes play a key role in anticancer immunity $(1,17)$, and a decreasing
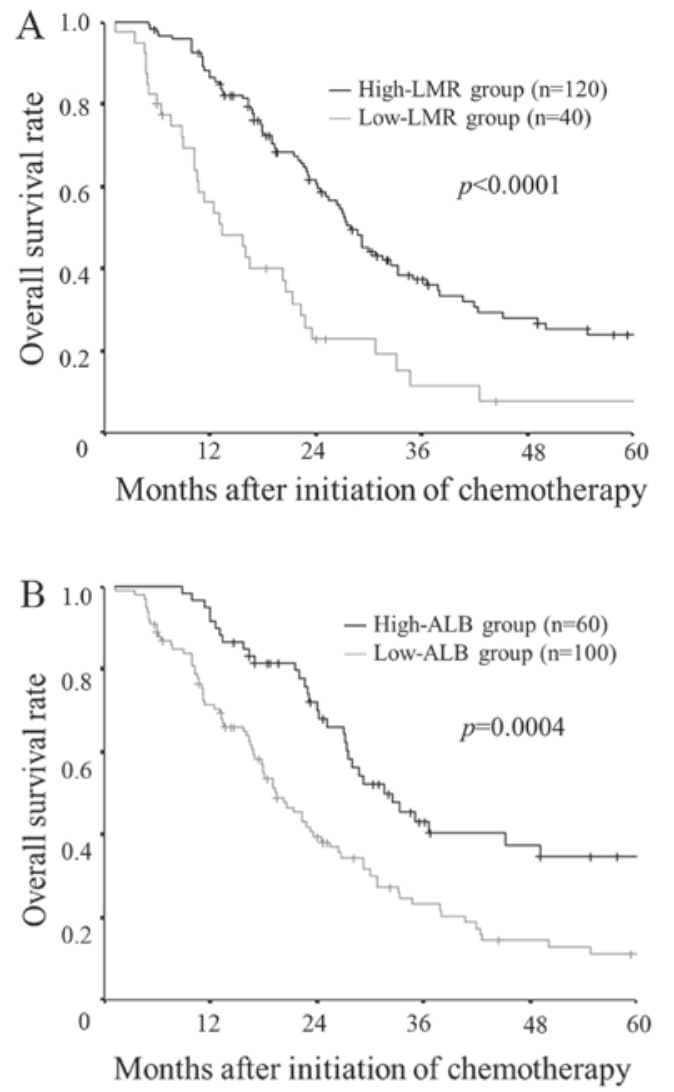

Figure 3. Kaplan-Meier survival curves for the overall survival according to the (A) LMR and (B) serum ALB. LMR, lymphocyte-to-monocyte ratio; ALB, albumin concentration

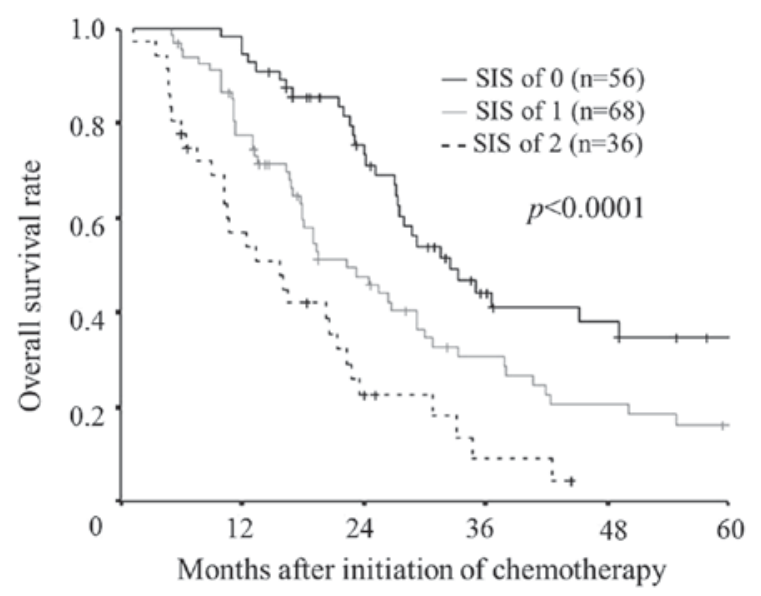

Figure 4. Kaplan-Meier survival curves for the overall survival according to the SIS based on the new cut-off values. SIS, systemic inflammatory score.

number of lymphocytes has been reported to be associated with a poor prognosis $(18,19)$. In contrast, monocytes contribute to cancer progression $(1,20,21)$. Circulating monocytes differentiate into macrophages in the cancer microenvironment $(22,23)$. Most macrophages in the cancer microenvironment have an M2-like phenotype and promote tumor growth, angiogenesis and metastasis $(20,24)$. Thus an increasing number of monocytes has been reported to be associated with a poor prognosis $(5,18,25)$. For these reasons, a low LMR is associated with a poor prognosis. 
The serum albumin concentration and white blood cell count are inexpensive to measure and are routinely applied in clinical practice. The combination of these two inflammatory markers based on different mechanisms may enable a more accurate prognostic prediction.

Both the serum albumin concentration and the LMR, which are components of the SIS, are markers related to inflammation, but their severity is not always correlated with each other (11). Therefore, the combination of these two markers enables a more detailed stratification. The SIS can be used to classify patients into three risk subgroups, whereas most inflammatory markers reported as prognostic markers in previous reports are only able to divide patients into two groups. The GPS as well as the SIS can classify patients into three risk subgroups. However, according to the GPS, most patients (80-90\%) are classified into the low-risk group $(10,26,27)$, and the distribution of the GPS score is not well-balanced. In contrast, the distribution of the SIS score is relatively well-balanced. These results suggest that the SIS may have higher clinical utility than other inflammatory markers.

According to the original cut-off values defined in a previous report, the SIS was significantly associated with the survival. However, the optimum cut-off values derived in our dataset was different from those obtained in the previous report. As cancer progresses, the degree of inflammation caused by the response of the host to the cancer increases (3). In previous reports, the inflammatory markers tended to increase as the stage progressed $(3,10,28)$. Furthermore, even at the same stage, the degree of inflammation may vary depending on the type of cancer. The optimum cut-off values of the inflammatory markers used in previous reports differed by type of cancer, even at the same stage (29-31). Therefore, it is necessary to reset the optimum cut-off value of the serum albumin concentration and the LMR, which is most closely associated with the prognosis, depending on the characteristics of the target, such as the cancer type and stage. The optimum cut-off value of the SIS needs to be examined in further studies, which include a large unified population of cancer types, stages and treatments. The same may be true of the cut-off for the GPS.

The AUC of the ROC curve for the LMR and the serum albumin concentration were relatively low, despite both markers having been reported to be useful prognostic markers in many previous reports $(5,6,16)$. We thought that the small number of cases was the reason for the low AUC. A large prospective study is therefore necessary to confirm the usefulness of the SIS as a prognostic marker.

In conclusion, the SIS is considered to be a useful biomarker for predicting the survival outcomes in patients with unresectable mCRC cancer who undergo chemotherapy, although the optimum cut-off value according to each patient's background needs to be examined in further studies. Patients with high SIS scores are expected to have a poor prognosis. Thus, an intensive chemotherapy regimen aiming at cytoreduction-as opposed to disease control-should be selected for patients with a high SIS score. The SIS may contribute to decisions regarding the choice of therapeutic strategies.

\section{Acknowledgements}

Not applicable.

\section{Funding}

No funding was received.

\section{Availability of data and materials}

The datasets used and/or analyzed during the current study are available from the corresponding author on reasonable request.

\section{Authors' contributions}

MS and KM designed the study, performed the statistical analysis and drafted the manuscript. HN, TF, SM, KK and RA collected the clinical data and critically revised the manuscript. KH and MO designed the study and critically reviewed the manuscript. All authors read and approved the final manuscript.

\section{Ethics approval and consent to participate}

All patients were informed of the investigational nature of this study and provided their written informed consent. Full ethical approval was granted by the ethics committee of Osaka City University (approval number 926).

\section{Consent for publication}

All patients provided written informed consent for the publication of their data.

\section{Competing interests}

The authors declare that they have no competing interests.

\section{References}

1. Mantovani A, Allavena P, Sica A and Balkwill F: Cancer-related inflammation. Nature 454: 436-444, 2008.

2. Balkwill $\mathrm{F}$ and Mantovani A: Inflammation and cancer: Back to Virchow? Lancet 357: 539-545, 2001.

3. Shibutani M, Maeda K, Nagahara H, Noda E, Ohtani H, Nishiguchi $\mathrm{Y}$ and Hirakawa K: A high preoperative neutrophil-to-lymphocyte ratio is associated with poor survival in patients with colorectal cancer. Anticancer Res 33: 3291-3294, 2013.

4. Absenger G, Szkandera J, Stotz M, Postlmayr U, Pichler M, Ress AL, Schaberl-Moser R, Loibner H, Samonigg H and Gerger A: Preoperative neutrophil-to-lymphocyte ratio predicts clinical outcome in patients with stage II and III colon cancer. Anticancer Res 33: 4591-4594, 2013.

5. Shibutani M, Maeda K, Nagahara H, Iseki Y, Ikeya T and Hirakawa K: Prognostic significance of the preoperative lymphocyte-to-monocyte ratio in patients with colorectal cancer. Oncol Lett 13: 1000-1006, 2017.

6. Guo YH, Sun HF, Zhang YB, Liao ZJ, Zhao L, Cui J, Wu T, Lu JR, Nan KJ and Wang SH: The clinical use of the platelet/lymphocyte ratio and lymphocyte/monocyte ratio as prognostic predictors in colorectal cancer: A meta-analysis. Oncotarget 8: 20011-20024, 2017.

7. Liu Y, He X, Pan J, Chen S and Wang L: Prognostic role of Glasgow prognostic score in patients with colorectal cancer: Evidence from population studies. Sci Rep 7: 6144, 2017.

8. Kishiki T, Masaki T, Matsuoka H, Kobayashi T, Suzuki Y, Abe N, Mori T and Sugiyama M: Modified Glasgow prognostic score in patients with incurable stage IV colorectal cancer. Am J Surg 206: 234-240, 2013. 
9. Chang Y, An H, Xu L, Zhu Y, Yang Y, Lin Z and Xu J: Systemic inflammation score predicts postoperative prognosis of patients with clear-cell renal cell carcinoma. Br J Cancer 113: 626-633, 2015.

10. Suzuki Y, Okabayashi K, Hasegawa H, Tsuruta M, Shigeta K, Kondo $\mathrm{T}$ and Kitagawa Y: Comparison of preoperative inflammation-based prognostic scores in patients with colorectal cancer. Ann Surg 267: 527-531, 2018.

11. Eltohami YI, Kao HK, Lao WW, Huang Y, Abdelrahman M, Liao CT, Yen TC and Chang KP: The prediction value of the systemic inflammation score for oral cavity squamous cell carcinoma. Otolaryngol Head Neck Surg: Jan 1, 2018 (Epub ahead of print).

12. Cassidy J, Clarke S, Díaz-Rubio E, Scheithauer W, Figer A, Wong R, Koski S, Lichinitser M, Yang TS, Rivera F, et al: Randomized phase III study of capecitabine plus oxaliplatin compared with fluorouracil/folinic acid plus oxaliplatin as first-line therapy for metastatic colorectal cancer. J Clin Oncol 26: 2006-2012, 2008.

13. Tournigand C, André T, Achille E, Lledo G, Flesh M, Mery-Mignard D, Quinaux E, Couteau C, Buyse M, Ganem G, et al: FOLFIRI followed by FOLFOX6 or the reverse sequence in advanced colorectal cancer: A randomized GERCOR study. J Clin Oncol 22: 229-237, 2004

14. Yamada Y, Takahari D, Matsumoto H, Baba H, Nakamura M, Yoshida K, Yoshida M, Iwamoto S, Shimada K, Komatsu Y, et al: Leucovorin, fluorouracil, and oxaliplatin plus bevacizumab versus S-1 and oxaliplatin plus bevacizumab in patients with metastatic colorectal cancer (SOFT): An open-label, non-inferiority, randomised phase 3 trial. Lancet Oncol 14: 1278-1286, 2013.

15. McMillan DC, Elahi MM, Sattar N, Angerson WJ, Johnstone J and McArdle CS: Measurement of the systemic inflammatory response predicts cancer-specific and non-cancer survival in patients with cancer. Nutr Cancer 41: 64-69, 2001.

16. Nazha B, Moussaly E, Zaarour M, Weerasinghe C and Azab B: Hypoalbuminemia in colorectal cancer prognosis: Nutritional marker or inflammatory surrogate? World J Gastrointest Surg 7: 370-377, 2015

17. Dunn GP, Old LJ and Schreiber RD: The immunobiology of cancer immunosurveillance and immunoediting. Immunity 21: 137-148, 2004

18. Shibutani M, Maeda K, Nagahara H, Ohtani H, Sakurai K, Yamazoe S, Kimura K, Toyokawa T, Amano R, Tanaka H, et al: Prognostic significance of the lymphocyte-to-monocyte ratio in patients with metastatic colorectal cancer. World J Gastroenterol 21: 9966-9973, 2015.

19. Cézé N, Thibault G, Goujon G, Viguier J, Watier H, Dorval E and Lecomte T: Pre-treatment lymphopenia as a prognostic biomarker in colorectal cancer patients receiving chemotherapy. Cancer Chemother Pharmacol 68: 1305-1313, 2011.
20. Condeelis J and Pollard JW: Macrophages: Obligate partners for tumor cell migration, invasion, and metastasis. Cell 124: 263-266, 2006.

21. Leek RD and Harris AL: Tumor-associated macrophages in breast cancer. J Mammary Gland Biol Neoplasia 7: 177-189, 2002.

22. Qian BZ and Pollard JW: Macrophage diversity enhances tumor progression and metastasis. Cell 141: 39-51, 2010.

23. Mantovani A, Bottazzi B, Colotta F, Sozzani S and Ruco L: The origin and function of tumor-associated macrophages. Immunol Today 13: 265-270, 1992.

24. Pollard JW: Tumour-educated macrophages promote tumour progression and metastasis. Nat Rev Cancer 4: 71-78, 2004.

25. Paik KY, Lee IK, Lee YS, Sung NY and Kwon TS: Clinical implications of systemic inflammatory response markers as independent prognostic factors in colorectal cancer patients. Cancer Res Treat 46: 65-73, 2014.

26. Sugimoto K, Komiyama H, Kojima Y, Goto M, Tomiki Y and Sakamoto K: Glasgow prognostic score as a prognostic factor in patients undergoing curative surgery for colorectal cancer. Dig Surg 29: 503-509, 2012.

27. Furukawa K, Shiba H, Haruki K, Fujiwara Y, Iida T, Mitsuyama Y, Ogawa M, Ishida Y, Misawa T and Yanaga K: The Glasgow prognostic score is valuable for colorectal cancer with both synchronous and metachronous unresectable liver metastases. Oncol Lett 4: 324-328, 2012.

28. Jia J, Zheng X, Chen Y, Wang L, Lin L, Ye X, Chen Y, Chen D and Dettke M: Stage-dependent changes of preoperative neutrophil to lymphocyte ratio and platelet to lymphocyte ratio in colorectal cancer. Tumour Biol 36: 9319-9325, 2015.

29. Shibutani M, Maeda K, Nagahara H, Ohtani H, Sakurai K, Yamazoe A, Kimura K, Toyokawa T, Amano R, Kubo N, et al: Significance of markers of systemic inflammation for predicting survival and chemotherapeutic outcomes and monitoring tumor progression in patients with unresectable metastatic colorectal cancer. Anticancer Res 35: 5037-5046, 2015.

30. Tanaka H, Muguruma K, Toyokawa T, Kubo N, Ohira M and Hirakawa K: Differential impact of the neutrophil-lymphocyte ratio on the survival of patients with stage IV gastric cancer. Dig Surg 31: 327-333, 2014.

31. Cedrés S, Torrejon D, Martínez A, Martinez P, Navarro A, Zamora E, Mulet-Margalef N and Felip E: Neutrophil to lymphocyte ratio (NLR) as an indicator of poor prognosis in stage IV non-small cell lung cancer. Clin Transl Oncol 14: 864-869, 2012. 